\title{
Isolamento e tipizzazione di Brucella melitensis biovar 3 da emocoltura, utilizzando il sistema di rilevazione microbica Bact/Alert
}

\author{
Maria Teresa Allù', Chiara Piraino², Francesca Messina², Franco Sciurba ${ }^{2}$ \\ 'Laboratorio Analisi Chimico-cliniche e Microbiologiche, Azienda Ospedaliera Ragusa \\ ${ }^{2}$ Istituto Zooprofilattico Sperimentale per la Sicilia, Palermo
}

Key words: Brucella melitensis, automated blood culture system, bacteriological tests

Detection and identification of Brucella melitensis biovar 3 from blood culture using the Bactec/Alert microbial detection system

\section{SUMMARY}

Brucella melitensis is the aetiologic agent of brucellosis in small ruminants (goats,sheeps). Humans are susceptible to $B$. melitensis, which gives a severe and long lasting form of the disease. Diagnosis needs to be supported by laboratory tests; blood culture is the standard method and is often effective during the acute phase. Development of automated blood culture systems and technical improvements have resulted in gradual increase in the sensitivity of methods and in shortening the detection time of Brucella spp The identification at the biovar level is performed by carbon dioxide requirement, production of hydrogen sulphide, urease activity, dye (thionin and basic fuchsin) sensitivity, lysis by phages, agglutination with monospecific $A$ and $M$ anti-sera.

\section{INTRODUZIONE}

La Brucellosi è una zoonosi tuttora assai diffusa nel mondo, causata da microorganismi appartenenti al genere Brucella. Brucella spp comprende coccobacilli Gram-negativi di piccole dimensioni $(0.5-0.6 \times 1.5 \mu \mathrm{m})$, patogeni intracellulari facoltativi (1). Tali microrganismi infettano principalmente bovini, ovini, caprini e altri mammiferi, nei quali causano aborto, morte del feto e infezioni dell'apparato riproduttore. Sulla base essenzialmente delle caratteristiche fenotipiche ed antigeniche sono state riconosciute sei specie: Brucella abortus (tipica dei bovini), Brucella melitensis (soprattutto in ovini e caprini), Brucella suis (in suini), Brucella canis (nel cane), Brucella ovis (in ovini) e Brucella neotomae (nei ratti) (4, 5). Recentemente sono state descritte altre due specie isolate da mammiferi marini: Brucella cetaceae e Brucella pinnipediae (2). Le specie che causano brucellosi nell'uomo sono, in ordine di incidenza e patogenicità: Brucella melitensis, Brucella abortus, Brucella suis e Brucella canis. Brucella melitensis è la specie più virulenta e maggiormente implicata nelle infezioni umane, provocando una grave malattia acuta con varie complicanze. Brucella melitensis è diffusa prevalentemente nelle regioni del bacino del Mediterraneo, nell'America latina e nel Medio Oriente.

Brucella spp è trasmessa all'uomo attraverso tre vie principali: il contatto diretto con tessuti animali infetti, l'ingestione di latte contaminato o di prodotti caseari non pastorizzati, l'inalazione di batteri attraverso l'aerosol. Brucella spp è in grado di eludere i meccanismi di difesa dell'ospite, di sopravvivere come organismo intracellulare nelle cellule fagocitiche ed è capace di causare una prolungata morbosità con recidive a distanza e cronicità. Brucella melitensis è la specie più virulenta in quanto possiede una maggiore capacità di resistere all'effetto battericida del siero e all'uccisione mediata dai fagociti. La diagnosi di brucellosi necessita ovviamente di essere supportata dai test specifici di laboratorio: l'emocoltura rappresenta il metodo standard ed è efficace durante la fase acuta di malattia, le indagini sierologiche (basate su reazioni di agglutinazione) oltre che in fase acuta sono utili nella diagnosi di forme subcliniche e croniche.

\section{DESCRIZIONE DEL CASO}

Un soggetto di 37 anni e di sesso maschile, che presenta febbre (oltre i $38^{\circ} \mathrm{C}$ ), dolore toracico ed esantema diffuso su tutto il corpo, viene ricoverato presso l'U.O. di Malattie infettive; qui viene sottoposto a prelievo venoso per esami ematochimici ed emocoltura: vengono eseguite tre emocolture (ad intervalli di 12 ore ciascuna) utilizzando per ciascuna due flaconi di Bact/Alert (un flacone per aerobi ed uno per anaerobi). Gli esami ematochimici evidenziano aumento della Proteina $\mathrm{C}$ reattiva $(25 \mathrm{mg} / \mathrm{l})$, un leggero movimento della VES ( $21 \mathrm{~mm}$ alla I ora) ed iposideremia $(\mathrm{Fe}=35$ 
$\mathrm{mcg} / \mathrm{dl})$. Dall'ecografia dell'addome risulta milza aumentata di volume mentre il fegato è nella norma. Viene instaurata una terapia antibiotica con teicoplanina $(0.4 \mathrm{~g} / \mathrm{die} \mathrm{x} 4 \mathrm{gg}$ e.v. $)$ e ciprofloxacina $(0.2 \mathrm{~g} /$ die $\mathrm{x} 4 \mathrm{gg}$ e.v.). Le condizioni cliniche non migliorano e viene instaurata una nuova terapia con levofloxacina ( $0.5 \mathrm{~g} /$ die $\mathrm{x} 4 \mathrm{gg}$ e.v.) e rifampicina ( $0.6 \mathrm{~g} /$ die x $4 \mathrm{gg}$ e.v.). Al quarto giorno viene isolato dall'emocoltura un ceppo batterico che viene identificato come Brucella spp.

Dopo comunicazione al reparto dell'isolamento di tale microrganismo, il paziente viene trattato con streptomicina ( $1 \mathrm{~g} /$ die x $26 \mathrm{gg}$ i.m.) e ambramicina ( $125 \mathrm{mg} / \mathrm{die}$ x $26 \mathrm{gg}$ per os). Dopo qualche giorno si osserva un miglioramento del quadro clinico e dopo cinque giorni di terapia di associazione il paziente viene dimesso con la prescrizione di continuare a casa la terapia con streptomicina e ambramicina per 21 giorni.

\section{Identificazione batteriologica e tipizzazione}

Brucella melitensis è stata isolata dopo quattro giorni di incubazione da un flacone per aerobi con il sistema di rilevazione microbica Bact/Alert (bioMérieux), il quale utilizza un sensore colorimetrico a luce riflessa per monitorare la presenza e la produzione di $\mathrm{CO}_{2}$ (prodotta dal metabolismo batterico) disciolta nel terreno di coltura: in altre parole, lo strumento rileva lo sviluppo batterico tramite il viraggio di colore dell'indicatore presente sul fondo dei flaconi, indotto dalla liberazione di $\mathrm{CO}_{2}$ da parte dei microrganismi. L'esame batterioscopico diretto, previa colorazione di Gram, evidenziò cocco-bacilli Gram-negativi di piccole dimensioni. Dal flacone positivo vennero eseguite delle sottocolture su agar-sangue, agarcioccolato e agar -McConkey incubate in aerobiosi a $37^{\circ} \mathrm{C}$. Dopo 48 ore di incubazione si osservò crescita soltanto sulle piastre di agar- sangue e agar-cioccolato. La colorazione di Gram delle colture mostrò cocco-bacilli Gram-negativi. Sulle colonie isolate vennero eseguiti i test dell'ossidasi, della catalasi e dell'ureasi che diedero tutti esito positivo. I risultati di tali test (morfologia microscopica dei microorganismi isolati, assenza di crescita su McConkey, test dell'ossidasi, della catalasi e dell'ureasi positivi), hanno portato pertanto ad un'identificazione presuntiva dell'isolato come appartenere al genere Brucella. Il ceppo venne quindi inviato al Laboratorio di Batteriologia di seconda Istanza dell'Istituto Zooprofilattico Sperimentale per la Sicilia di Palermo, per con fermare l'identificazione a livello di genere e per effettuare la tipizzazione di specie e biovar di appartenenza (test biochimici e sierologici nonché fagotipizzazione) (tabella 1).

Tabella I. Prove di siero e biotipizzazione di specie di Brucella spp.

\begin{tabular}{|c|c|c|c|c|c|c|c|c|c|c|c|c|c|}
\hline & $M C$ & OX & CAT & MOB & URE & OF & $\mathrm{H} 2 \mathrm{~S}$ & $\begin{array}{l}\text { TH } \\
50\end{array}$ & \begin{tabular}{|l|} 
FU \\
50
\end{tabular} & \begin{tabular}{|l|} 
FU \\
100 \\
\end{tabular} & $\mathrm{O} 2$ & $A$ & $M$ \\
\hline $\mathrm{BMI}$ & - & + & + & - & + & - & - & + & + & + & + & - & + \\
\hline BM2 & - & + & + & - & + & - & - & + & + & + & + & + & - \\
\hline BM3 & - & + & + & - & + & - & - & + & + & + & + & + & + \\
\hline $\mathrm{BAI}$ & - & + & + & - & + & - & + & - & + & + & V & + & - \\
\hline BA2 & - & + & + & - & + & - & + & - & - & - & - & + & - \\
\hline $\mathrm{BA3}$ & - & + & + & - & + & - & + & + & + & + & $\mathrm{V}$ & + & - \\
\hline BA4 & - & + & + & - & + & - & + & - & + & + & V & - & + \\
\hline BA5 & - & + & + & - & + & - & - & + & + & + & + & - & + \\
\hline BA6 & - & + & + & - & + & - & V & + & + & + & + & + & - \\
\hline BA7 & - & + & + & - & + & - & V & + & + & + & + & + & + \\
\hline BA9 & - & + & + & - & + & - & + & + & + & + & $\mathrm{V}$ & - & + \\
\hline $\mathrm{BSI}$ & - & + & + & - & + & - & + & + & - & - & + & + & - \\
\hline $\mathrm{BS} 2$ & - & + & + & - & + & - & - & + & - & - & + & + & - \\
\hline BS3 & - & + & + & - & + & - & - & + & + & + & + & + & - \\
\hline BS4 & - & + & + & - & + & - & - & + & + & + & + & + & + \\
\hline $\mathrm{BN}$ & - & + & + & - & + & - & + & - & - & - & + & + & - \\
\hline $\mathrm{BO}$ & - & - & + & - & - & - & - & + & + & + & - & - & - \\
\hline$B C$ & - & + & + & - & + & - & - & + & - & + & - & - & - \\
\hline
\end{tabular}

BMI: Brucella melitensis biovar I; BM2: B. melitensis biovar2; BM3: B. melitensis biovar3; BAI: Brucella abortus biovar I; BA2: $B$. abortus biovar2; BA3: B. abortus biovar3; BA4: B. abortus biovar4; BA5: B. abortus biovar 5; BA6: B. abortus biovar6; BA7: B. abortus biovar 7; BA9: B. abortus biovar9; BSI: Brucella suis biovar I; BS2: B. suis biovar2; BS3: B. suis biovar3; BS4: B. suis biovar4; BN: Brucella neotomae; BO: Brucella ovis; BC: Brucella canis; MC: MacConkey agar; OX: Ossidasi; CAT: Catalasi; MOB: Mobilità; URE: Urea indolo medium; OF: O F medium; H2S: Idrogeno solforato test; TH 50:Tionina 1:50.000; FU 50: Fucsina 1:50.000; A: Antisiero monospecifico "A"; M:Antisiero monospecifico "M" 
Per la determinazione di specie sono state eseguite le seguenti prove: test dell'ureasi, test dell'H2S, test batteriostatici d'inibizione di crescita ad opera dei coloranti tionina e fuxina, prove di tipizzazione fagica (utilizzando tre batteriofagi: Weibridge, Iztnagar, Tblisi) e prove sierologiche (utilizzando antisieri monospecifici A ed M).Correlando i dati biochimici con quelli fagici e sierologici, il microrganismo isolato è stato identificato come Brucella melitensis biovar 3.

\section{COMMENTO}

La brucellosi è un infezione sistemica che può interessare vari organi del corpo e i casi in cui le manifestazioni gastrointestinali, respiratorie, cutanee e neurologiche predominano non sono insoliti (8). A causa dell'ampio spettro di manifestazioni cliniche, la brucellosi può mimare altre infezioni e condizioni non infettive, per cui la diagnosi della malattia può essere ritardata o difficile (11). Attualmente la diagnosi di brucellosi si basa principalmente sull'esame sierologico (sieroagglutinazione rapida e fissazione del complemento) e sull'isolamento di Brucella spp dal sangue (10). Recentemente, per l'identificazione dei microrganismi appartenenti al genere Brucella, sono stati sviluppati dei metodi di diagnostica molecolare basati sull'amplificazione di differenti targets genomici mediante l'uso della PCR (2). I recenti sviluppi sull'automazione dei sistemi di emocoltura hanno enormemente diminuito il tempo richiesto per l'isolamento di microorganismi a lenta crescita. Casas, et al. hanno studiato l'appropriatezza del sistema Bact/Alert per la ricerca in vitro di Brucella spp riportando un tempo medio per l'isolamento di 2-3 giorni (3); è consigliabile sospettare la presenza di Brucella spp quando la positività è rilevata solo dai flaconi per aerobi dopo 2-3 giorni di incubazione. Il personale che potenzialmente potrebbe venire a contatto con germi ascrivibili al genere Brucella deve sapere che questo è un microrganismo estremamente contagioso e che richiede un livello di sicurezza 2 per i laboratori dove viene maneggiato il sangue del paziente ed un livello 3 per quelli dove si effettua la coltura dei microorganismi. Il caso clinico riportato mostra che, attraverso l'emocoltura e la successiva identificazione del germe in causa, è stato possibile effettuare una corretta diagnosi ed uno specifico trattamento terapeutico.

\section{BIBLIOGRAFIA}

1. Alton GG, Jones LM, Pietz DE. Laboratory techniques in brucellosis, $2^{\text {nd }} \mathrm{ed}$. World health Organization, Geneva, 1975.

2. Bricker BJ, Ewalt DR, MacMillan AP, et al. Molecular characterization of Brucella strains isola- ted from marine mammals. J Clin Microbiol 2000; 38 : 1258-62.

3. Casas J, Portal Y, Llosà J, et al. Detection of Brucella by an automatic bloodculture system: Bact/Alert. Emferm Infecc Microbiol Clin 1994; 12: 497-500.

4. Corbel MJ. Brucellosis: an overview. Emerg Infect Dis 1997; 3: 213-21.

5. Moreno E, Cloeckaert A, Moriyon I. Brucella evolution and taxonomy. Vet Microbiol 2002; 90: 209-27.

6. Rodriguez-Torres A, Fermoso J, Landinez R. Brucellosis. Medicine 1983; 48: 3126-36.

7. Ruiz J, Lorente I, Perez J, et al. Diagnosis of brucellosis by using blood cultures. J Clin Microbiol 1997; 35: 2417-8.

8. Santini C, Baiocchi P, Berardelli A, et al. A case of brain abscess due to Brucella melitensis. Clin Infect Dis 1994; 19: 977-8.

9. Weyant RS, Moss CW, Weaver RE, et al. Identification of unusual pathogenic Gram-negative aerobic and facultatively anaerobic bacteria, $2^{\text {nd }}$ ed. Williams \& Wilkins, Baltimore Md, 1995.

10. Yagupsky P. Detection of Brucellae in blood cultures. J Clin Microbiol 1999; 37: 3437-42.

11. Young EJ. An overview of human brucellosis. Clin Infect Dis 1995; 21: 283-90.
Maria Teresa Allù

Laboratorio di analisi chimico-cliniche e microbiologiche

Azienda Ospedaliera Ragusa

P.zza Ospedale Civile 1 - 97100 Ragusa

Tel.0932 600148

E-mail: mariateresa.allu@virgilio.it 